\title{
Epitaxial and layer-by-layer growth of EuO thin films on yttria-stabilized cubic zirconia (001) using MBE distillation
}

\author{
R. Sutarto, ${ }^{1}$ S. G. Altendorf, ${ }^{1}$ B. Coloru, ${ }^{1}$ M. Moretti Sala,${ }^{1}$ T. Haupricht,${ }^{1}$ C. F. Chang,,${ }^{1}$ Z. Hu, ${ }^{1}$ C. \\ Schüßler-Langeheine, ${ }^{1}$ N. Hollmann, ${ }^{1}$ H. Kierspel,${ }^{1}$ H. H. Hsieh, ${ }^{2}$ H.-J. Lin,${ }^{3}$ C. T. Chen,${ }^{3}$ and L. H. Tjeng ${ }^{1}$ \\ 1 II. Physikalisches Institut, Universität zu Köln, Zülpicher Str. 77, 50937 Köln, Germany \\ ${ }^{2}$ Chung Cheng Institute of Technology, National Defense University, Taoyuan 335, Taiwan \\ ${ }^{3}$ National Synchrotron Radiation Research Center, 101 Hsin-Ann Road, Hsinchu 30077, Taiwan
}

(Dated: November 3, 2018)

\begin{abstract}
We have succeeded in growing epitaxial and highly stoichiometric films of EuO on yttria-stabilized cubic zirconia (YSZ) (001). The use of the Eu-distillation process during the molecular beam epitaxy assisted growth enables the consistent achievement of stoichiometry. We have also succeeded in growing the films in a layer-by-layer fashion by fine tuning the Eu vs. oxygen deposition rates. The initial stages of growth involve the limited supply of oxygen from the YSZ substrate, but the EuO stoichiometry can still be well maintained. The films grown were sufficiently smooth so that the capping with a thin layer of aluminum was leak tight and enabled ex situ experiments free from trivalent Eu species. The findings were used to obtain recipes for better epitaxial growth of $\mathrm{EuO}$ on $\mathrm{MgO}$ (001).

PACS numbers: 68.55.-a, 75.70.Ak, 78.70.Dm, 79.60.Dp, 81.15.Hi
\end{abstract}

\section{INTRODUCTION}

Stoichiometric $\mathrm{EuO}$ is a ferromagnetic semiconductor with a Curie temperature $\left(T_{C}\right)$ of $69 \mathrm{~K}$ and a band gap of about $1.2 \mathrm{eV}$ at room temperature $\underline{1}$ Upon electron doping, the material shows a wealth of spectacular phenomena, including a metal-to-insulator transition and colossal magnetoresistance, where the change in resistivity can exceed 8-10 orders of magnitude. ${ }^{2.3 .4}$ The Curie temperature can also be doubled by electron doping $\frac{5}{5}$ and even almost tripled by pressure $\frac{6.7}{\underline{m}}$ In the ferromagnetic state the conduction band shows a splitting of about $0.6 \mathrm{eV}$ between the spin-up and spin-down states leading to an almost $100 \%$ spin polarization of the charge carriers in electron doped $\mathrm{EuO} \stackrel{\underline{8}}{\text { Th }}$ These properties make $\mathrm{EuO}$ a very attractive candidate for fundamental research in the field of spintronics.

$\mathrm{EuO}$ in thin film form has been studied already in the late 1960s and early 1970s. Use was made of coevapora-

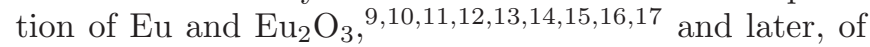
evaporation of $\mathrm{Eu}$ in an oxygen atmosphere $\underline{18,19,20,21,22}$ all under technical vacuum conditions, i.e., pressures in the range of $10^{-6}-10^{-5}$ mbar. The preparation of thin films was considered as a convenient alternative synthesis route for $\mathrm{EuO}$, alternative to that of the bulk synthesis which required very high temperatures with a delicate phase diagram. ${ }^{23}$ The use of the thinfilm preparation route specifically facilitated doping dependence studies using rare-earth and transition-metal impurities, $10,13,14,15,16$

After a pause of two decades, a strong renewed interest in $\mathrm{EuO}$ thin films emerged in recent years. This time it is the thin-film community which is making the attempt to utilize the extraordinary properties of $\mathrm{EuO}$ for device applications $\frac{8,24,25,26,27,28,29,30,31,32,33,34,35,36,37,38,39,40,41}{4}$ Part of the motivation also originates from the fact that tremendous progress has been made in preparation technologies, e.g., molecular beam epitaxy (MBE) under ultra-high-vacuum conditions, and that new analysis methods have become available, e.g., synchrotron-based spectroscopies. All in all, these new efforts have culminated in a high point such that $\mathrm{EuO}$ thin films can be grown epitaxially on $\mathrm{Si}$, demonstrating its potential for spintronics applications $\underline{\underline{36}}$

It is remarkable in the recent $\mathrm{EuO}$ research $24,25,26,27,29,30,31,32,34,35,36,37,38,39,40$ that control of the stoichiometry is nevertheless still a serious issue. Many studies reported that $\mathrm{Eu}^{3+}$ ions were present in their films and/or that the magnetic moment per f.u. was not close to the expected $7 \mu_{B}$ for a $4 f^{7}$ system. It is not clear in what precision the relative supply rates of oxygen and europium were controlled in these works. We will show below that this control need not be precise as long as one is in the $\mathrm{so}^{-}$-called $\mathrm{Eu}$-distillation condition during growth.

Also layer-by-layer growth has - to our knowledge never been mentioned, although epitaxy has been often reported. We therefore set out to do a renewed growth study. We have chosen for yttria-stabilized cubic zirco-

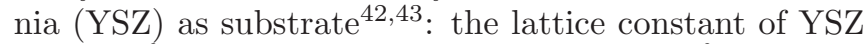
is $5.142 \AA$, practically identical to the $5.144 \AA$ value for $\mathrm{EuO}$ at room temperature, $\underline{44}$ and epitaxy has been reported already $\stackrel{24,28,36}{=}$ Yet, it was also claimed that con-

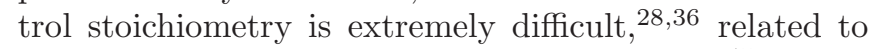
the fact that YSZ acts as a source of oxygen $\frac{45}{}$ during the MBE growth process. We will show below that we do have achieved full control of the growth process, i.e., with stoichiometry control and layer-by-layer epitaxial growth, and that we can use these results as a firm basis for further studies including the doping dependence and the specific use of well-defined interfaces. 

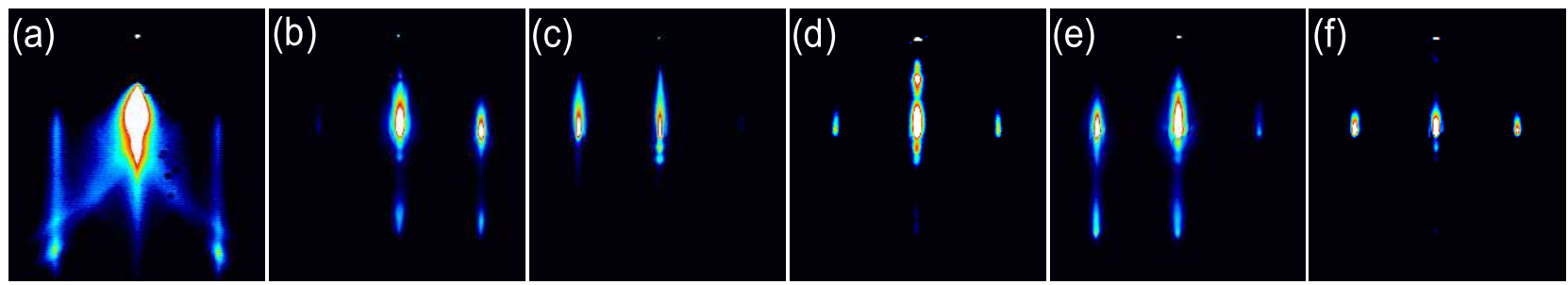

FIG. 1: (Color online) RHEED photographs of (a) clean and annealed YSZ (001), [(b)-(d)] EuO films on YSZ (001) after 10 min of growth at $400^{\circ} \mathrm{C}$ with $8.1-8.2 \AA /$ min Eu flux rates, and [(e)-(f)] with $4.2-4.3 \AA / m i n$ Eu flux rates. The oxygen pressure in the chamber was $4 \times 10^{-8}$ mbar for (b), and $2 \times 10^{-8}$ mbar for (c) and (e). No oxygen was supplied into the chamber for (d) and (f). The RHEED electron energy was $20 \mathrm{keV}$ with the beam incident along the [100] direction.

\section{EXPERIMENT}

The EuO films were grown in an ultra-high-vacuum MBE facility with a base pressure of $2 \times 10^{-10} \mathrm{mbar}$, maintained by a cryopump. High purity Eu metal from AMES Laboratory was evaporated from an EPI effusion cell with a BN crucible at temperatures between $460^{\circ} \mathrm{C}$ and $525^{\circ} \mathrm{C}$. Proper degassing of the Eu material (mostly hydrogen gas) ensured that during Eu evaporation the pressure was kept below $3 \times 10^{-9}$ mbar. The Eu deposition rate (4-8 $\AA / \mathrm{min})$ was calibrated using a quartzcrystal monitor which was moved to the sample growth position prior and after each growth. Molecular oxygen was supplied through a leak valve, and its pressure (4$16 \times 10^{-8}$ mbar) was monitored using an ion-gauge and a mass-spectrometer. The growth was terminated by closing first the oxygen leak valve and then the Eu shutter after $30 \mathrm{~s}$.

As substrates we used epipolished single crystals of YSZ from SurfaceNet GmbH and cleaved single crystals of $\mathrm{MgO}$ from TBL-Kelpin. The surface normal of the substrates are all the (001). The lattice constant of YSZ is $5.142 \AA$, very close to the $5.144 \AA$ value for $\mathrm{EuO}$ at room temperature. The lattice constant of $\mathrm{MgO}$ is 4.21 $\AA .44$ Prior to growth the substrates were annealed in situ at $T=600^{\circ} \mathrm{C}$ in an oxygen atmosphere of $5 \times 10^{-7} \mathrm{mbar}$ for at least $120 \mathrm{~min}$ in the case of YSZ, and in $1 \times 10^{-7}$ mbar for at least $60 \mathrm{~min}$ for $\mathrm{MgO}$, in order to obtain clean and well-ordered substrate surfaces. The substrates were kept at $T=400^{\circ} \mathrm{C}$ during growth.

The MBE facility is supplied with the EK-35-R reflection high-energy electron diffraction (RHEED) system from STAIB Instruments for in situ and online monitoring of the growth. The MBE facility is attached to an ultra-high-vacuum $\mu$-metal photoemission chamber equipped with a Scienta SES-100 electron energy analyzer and a Vacuum Generators twin crystal monochromatized $\mathrm{Al}-K_{\alpha}(h \nu=1486.6 \mathrm{eV})$ source for in situ $\mathrm{x}$-ray photoelectron spectroscopic (XPS) analysis. The overall energy resolution was set to $0.35 \mathrm{eV}$ and the Fermi level $E_{F}$ was calibrated using a polycrystalline Au reference. The $\mu$-metal chamber is also equipped with a Vacuum Generators Scientific T191 rear-view low-energy electron diffraction (LEED) system for further in situ structural characterization. The base pressure of the $\mu-$ metal chamber is $1 \times 10^{-10}$ mbar, and all characterizations herein were carried out at room temperature.

The MBE facility is also connected to a separate ultrahigh-vacuum chamber for the evaporation of aluminum as protective capping layer of the air-sensitive EuO films. This allows the ex situ characterizations using $\mathrm{x}$-ray reflectivity (XRR), superconducting quantum interference device (SQUID), and $\mathrm{x}$-ray absorption spectroscopy (XAS). The thickness of the aluminum capping is about 20-40 A. The XRR measurements were carried out using a Siemens D5000 diffractometer. The magnetic properties of the films were determined using a Quantum Design MPMS-XL7 SQUID magnetometer. The XAS measurements were performed at the Dragon beamline of the National Synchrotron Radiation Research Center (NSRRC) in Taiwan. The spectra were recorded using the totalelectron-yield method and the photon-energy resolution at the $\mathrm{Eu} M_{4,5}$ edges $(h \nu \approx 1100-1180 \mathrm{eV})$ was set at $\approx$ $0.6 \mathrm{eV}$.

\section{INITIAL STAGES OF GROWTH ON YSZ}

Figure 1(a) shows the RHEED photograph of the clean and annealed YSZ (001) before growth, and Figs. 1(b)1 (e) show the photographs after $10 \mathrm{~min}$ of $\mathrm{EuO}$ growth. The Eu flux rates were $8.1-8.2 \AA / m i n$ for (b)-(d) and 4.2-4.3 $\AA / \mathrm{min}$ for (e)-(f). The oxygen pressure in the chamber was $4 \times 10^{-8}$ mbar for (b) and $2 \times 10^{-8} \mathrm{mbar}$ for (c) and (e). No oxygen was supplied into the chamber for $(\mathrm{d})$ and (f). The YSZ substrate temperature was kept at $T=400^{\circ} \mathrm{C}$ during growth. The important result is that the general features of the RHEED patterns did not change during growth and that they are very similar to those of the clean YSZ for all Eu and O growth conditions. The distance between the streaks of the EuO films is identical to that of the pure YSZ, confirming that the in-plane lattice constants of EuO and YSZ are very closely matched.

Figure 2 shows the time dependence of the RHEED intensity of the specularly reflected beam during the EuO growth. We can clearly observe oscillations which are 


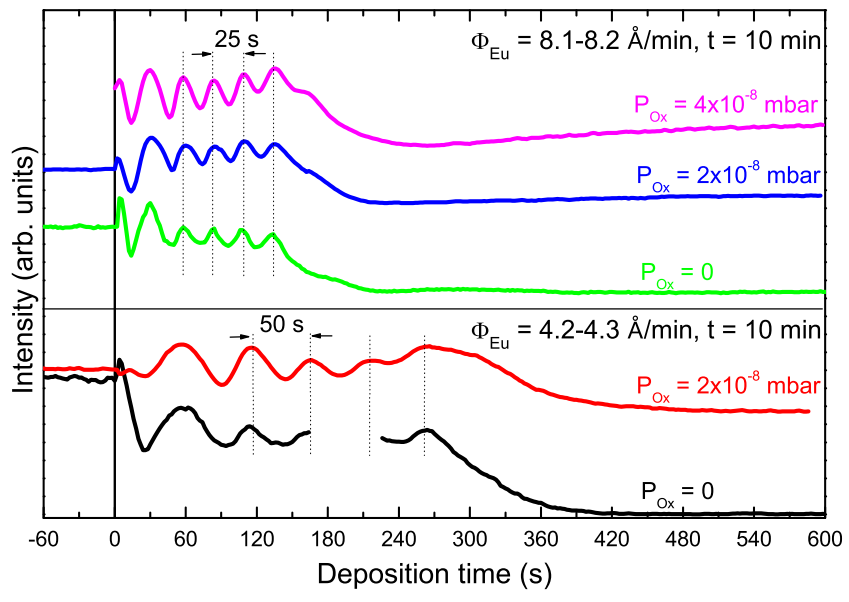

FIG. 2: (Color online) RHEED intensity oscillations of the specularly reflected electron beam, recorded during the deposition of EuO films on YSZ (001) using oxygen pressures $\left(P_{\mathrm{Ox}}\right)$ and Eu flux rates $\left(\Phi_{\mathrm{Eu}}\right)$ as indicated. The corresponding RHEED photographs after $10 \mathrm{~min}$ of growth are displayed in Figs. 1(b)-1(f).

indicative for a two-dimensional (2D) layer-by-layer or Frank-van der Merwe growth mode. It is surprising that there are only five to six oscillations for all deposition conditions as indicated in Fig. 2 and that these oscillations even exist in the absence of oxygen in the MBE chamber. It is important to note that the oscillation period does not depend on the oxygen pressure $P_{\mathrm{Ox}}$, thus also in the case of no oxygen in the chamber. This indicates that the oxygen needed for the formation of $\mathrm{EuO}$ must also come from the YSZ substrate. The $T=400^{\circ} \mathrm{C}$ substrate temperature apparently provides sufficient mobility for the oxygen ions to migrate to form at least five or six $\mathrm{EuO}$ layers. The oscillation period, which represents a formation of a new atomic single layer, is determined only by the Eu flux rate $\Phi_{\mathrm{Eu}}$ : reducing it by a factor of 2 , from 8.1-8.2 to $4.2-4.3 \AA / \mathrm{min}$, doubles the period, from 25 to $50 \mathrm{~s}$.

LEED photographs for all these films displayed a good single crystallinity. Figure 3 depicts examples for the case of no oxygen in the MBE chamber during growth. Also here we can observe a perfect (001) surface of the EuO rock-salt structure, consistent with the RHEED results. The LEED photographs were taken at electron beam energies of 213-215 eV since lower energies did not provide stable patterns due to charging.

To investigate the implications of observing only five to six oscillations, we also carried out photoemission experiments on those films. Figure 4, left panel (a), shows the $\mathrm{Zr} 3 d$ and $\mathrm{Eu} 4 d$ core level XPS spectra which were collected at normal emission. It can be clearly seen that the Zr signal is reduced when comparing the clean YSZ (bottom curve - black) with the EuO-covered YSZ (top three curves - blue, green, and red). The EuO films here were grown without oxygen in the MBE chamber. Different $\mathrm{Eu}$ flux rates and total time of growth are indicated
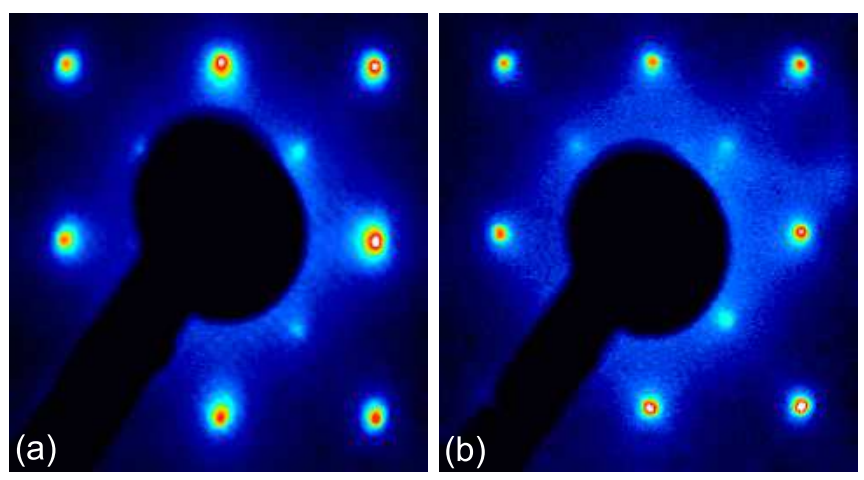

FIG. 3: (Color online) LEED photographs of epitaxial EuO films on YSZ substrate, grown for $10 \mathrm{~min}$ at $400^{\circ} \mathrm{C}$ in the absence of oxygen in the MBE chamber using (a) a $8.2 \AA / \mathrm{min}$ $\mathrm{Eu}$ flux rate and recorded at electron beam energy of $215 \mathrm{eV}$, and (b) a $4.3 \AA / \mathrm{min}$ Eu flux rate and recorded at electron beam energy of $213 \mathrm{eV}$.

in the figure. It is remarkable that the EuO-covered YSZ spectra have very similar Zr signals, and also equal Eu intensity, despite the fact that the total amount of Eu exposure is twice as large in the two middle curves (green and red) than in the top one (blue). This indicates that in the absence of oxygen in the MBE chamber, the growth of EuO is limited to five to six monolayers only and that the rest of the deposited Eu metal is re-evaporated back into the vacuum (the substrate temperature is $400^{\circ} \mathrm{C}$ ). In other words, the sticking coefficient for Eu after the completion of five to six monolayers is reduced to zero, suggesting that oxygen transport through $\mathrm{EuO}$ is much more difficult than in YSZ.

It is also important to investigate the chemical state of the Eu. Figure 4, right panel (b), depicts the O $2 p$ and $\mathrm{Eu} 4 f$ valence band spectra together with the $\mathrm{Zr} 4 p$, O $2 s$, and $\mathrm{Eu} 5 p$ core levels. The Eu $4 f$ lineshape in all the films is very characteristic for a $\mathrm{Eu}^{2+}$ system. The multiplet structure typical for $\mathrm{Eu}^{3+}$ is not visible. One can also observe that the $\mathrm{O} 2 p$ spectrum at $6-10 \mathrm{eV}$ binding energy for YSZ is converted into the $\mathrm{O} 2 p$ valence band at 4-7 eV typical for $\mathrm{EuO} \stackrel{8}{=}$ All these demonstrate that only $\mathrm{EuO}$ has been formed, free from $\mathrm{Eu}_{2} \mathrm{O}_{3}$ or $\mathrm{Eu}_{3} \mathrm{O}_{4}$ contaminants. This also means that YSZ can only oxidize Eu into the $2+$ state, and definitely not into the $3+$.

We have also carried out take-off angle-dependent XPS experiments on the films. Figure 5 shows the Zr $3 d$ and Eu $4 d$ core level XPS spectra of one of the EuO films of Fig. 4 (a) collected at grazing emission, i.e., $\Theta=70^{\circ}$ with respect to the surface normal, and at normal emission. One can clearly see that the Eu signal is not significantly reduced but the Zr signal has almost disappeared in the grazing-emission geometry. Since grazing emission means more surface sensitivity, this result confirms not only that the EuO film is on top of the YSZ substrate with negligible intermixing of the cations but also that the film is closed and flat.

Figure 6 depicts the $\mathrm{Zr} 3 d$ and $\mathrm{Eu} 4 d$ core level spectra 

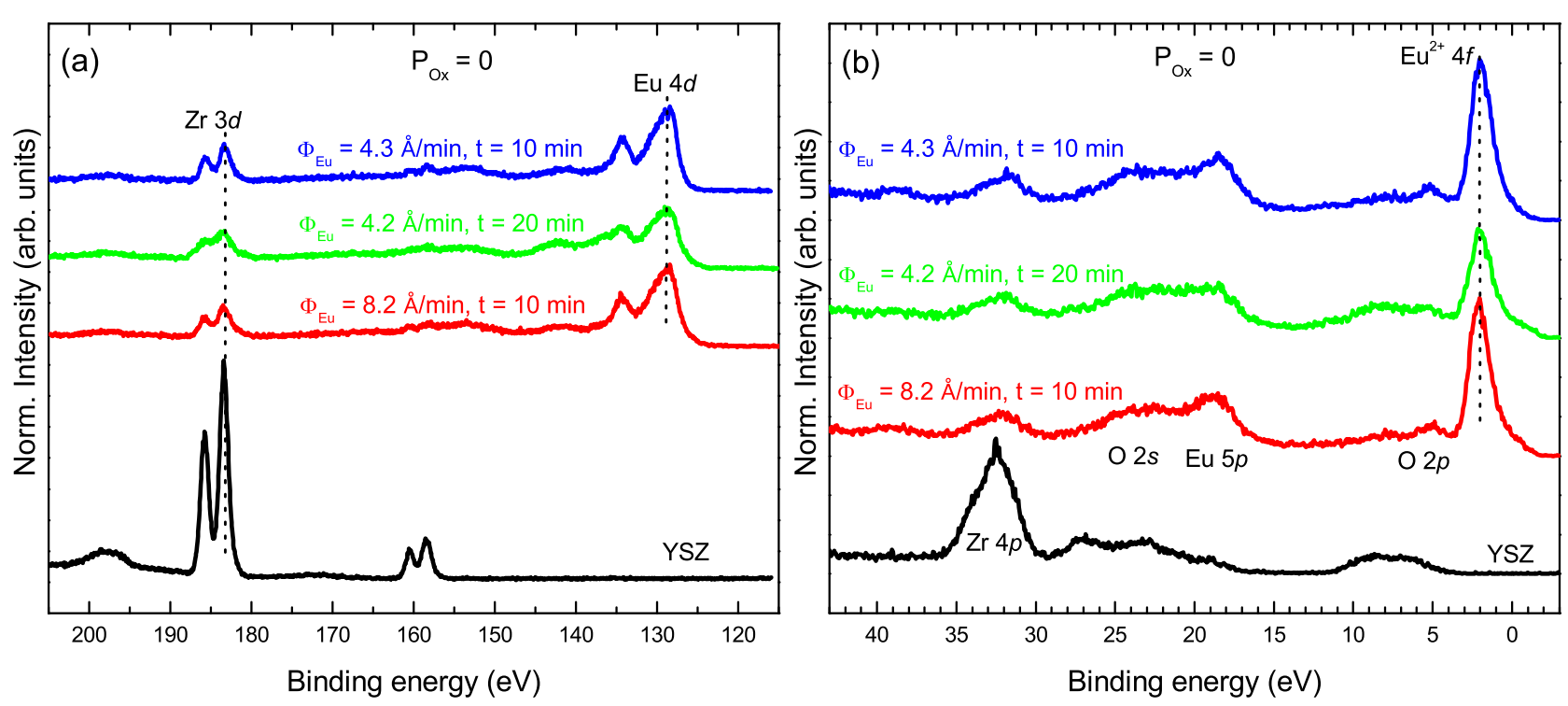

FIG. 4: (Color online) (a) Zr $3 d-$ Eu $4 d$ core level XPS spectra and (b) Zr $4 p-$ O $2 s-$ Eu $5 p$ core level and O $2 p-$ Eu $4 f$ valence band XPS spectra of EuO films on YSZ (001), grown at $400^{\circ} \mathrm{C}$ in the absence of oxygen in the MBE chamber. The spectra were collected at normal emission. From top to bottom: EuO film after $10 \mathrm{~min}$ of growth using a $4.3 \AA / \mathrm{min}$ Eu flux rate, after $20 \mathrm{~min}$ using $4.2 \AA / \mathrm{min}$, after $10 \mathrm{~min}$ using $8.2 \AA / \mathrm{min}$, and clean YSZ substrate.

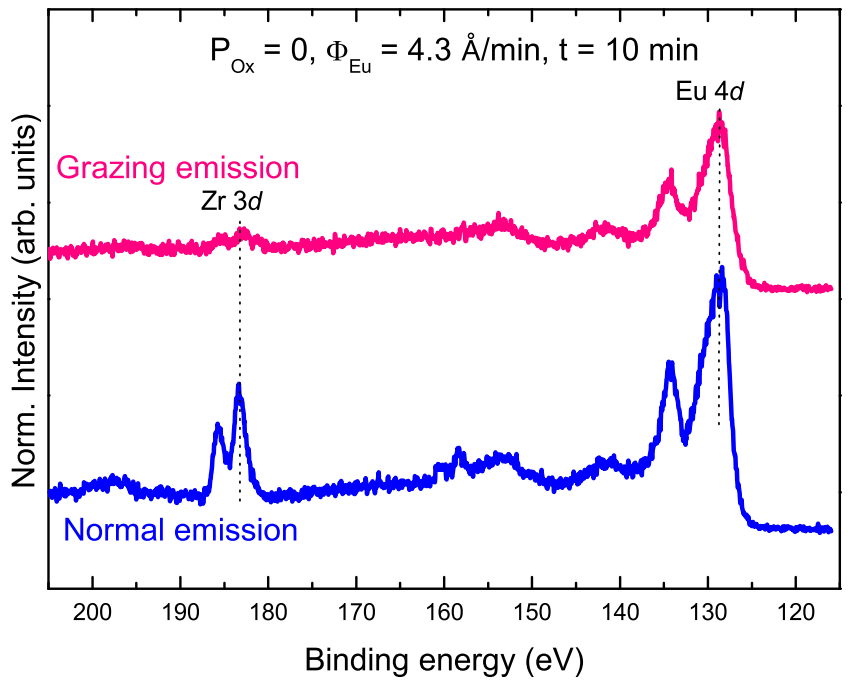

FIG. 5: (Color online) Take-off angle dependence of the Zr $3 d-$ Eu $4 d$ core level XPS spectra of a EuO film on YSZ (001). Top: grazing emission, i.e., $\Theta=70^{\circ}$ with respect to the surface normal. Bottom: normal emission. The film was grown at $400^{\circ} \mathrm{C}$ for 10 min with a $4.3 \AA / m i n$ Eu flux rate in the absence of oxygen in the MBE chamber.

(left panel), and the $\mathrm{Zr} 4 p$, O $2 s$, and Eu $5 p$ core level together with $\mathrm{O} 2 p$ and Eu $4 f$ valence band spectra (right panel) of EuO films grown with the supply of oxygen in the MBE chamber. Various oxygen pressures have been used as indicated in the figure. The Eu flux rate and the deposition time are identical for these films. One can clearly observe that the $\mathrm{Zr} 3 d$ signal is getting smaller when the oxygen pressure is increased, indicating that the thickness of the EuO film becomes larger. The lineshapes of the $\mathrm{Eu} 4 d$ and $\mathrm{Eu} 4 f$ levels are those of divalent $\mathrm{Eu}$ for pressures up to $12 \times 10^{-8}$ mbar. For a pressure of $12 \times 10^{-8}$ mbar or higher, however, the Eu $4 d$ and Eu $4 f$ spectral shapes start to change and show characteristics which indicate the presence of trivalent Eu. Apparently, for a Eu flux rate of $8.2 \AA / \mathrm{min}$ and substrate temperature of $400^{\circ} \mathrm{C}, 10-12 \times 10^{-8}$ mbar is the critical oxygen pressure below which $\mathrm{EuO}$ films can be made on YSZ (001) free from any $\mathrm{Eu}_{2} \mathrm{O}_{3}$ or $\mathrm{Eu}_{3} \mathrm{O}_{4}$ type of impurity phases.

We have also measured the photoemission spectra of these $\mathrm{EuO}$ films under grazing take-off angle conditions. Again, the Eu $4 d$ core level and Eu $4 f$ valence band spectra are all $2+$ as long as the oxygen pressures in the MBE chamber are below the critical $10-12 \times 10^{-8}$ mbar value. One example is shown in Fig. 7, where an oxygen pressure of $4 \times 10^{-8}$ mbar was used. This figure demonstrates that also the surface region of the films is free from $\mathrm{Eu}^{3+}$ species. It is interesting to compare the grazing with the normal emission spectra and also with the spectra displayed in Fig. 5. One can clearly see in Fig. 7 that the $\mathrm{Zr} 3 d$ signal is very much suppressed in grazing emission, even more suppressed than in the grazing emission spectrum of Fig. 5. This shows that the EuO films grown with the supply of oxygen in the MBE chamber are thicker, a not so surprising and yet very consistent observation since without oxygen we have found that the $\mathrm{EuO}$ film growth is limited to five to six monolayers.

The following picture can now be drawn about the initial stages of growth of EuO film on YSZ (001). In case there is no oxygen in the MBE chamber, YSZ is supplying all the oxygen required to form $\mathrm{EuO}$. The film is per- 

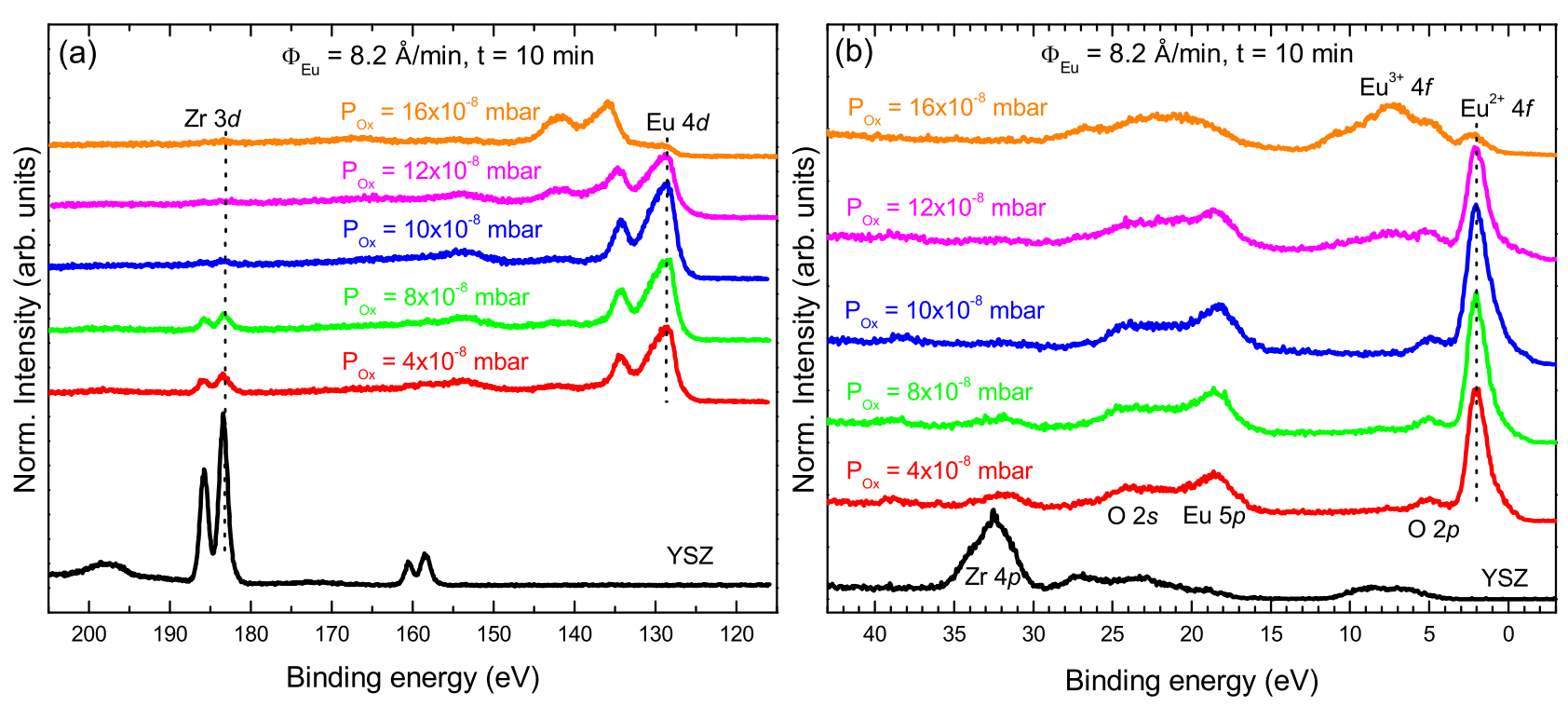

FIG. 6: (Color online) (a) Zr $3 d-\mathrm{Eu} 4 d$ core level XPS spectra and (b) Zr $4 p-\mathrm{O} 2 s-\mathrm{Eu} 5 p$ core level and O $2 p-$ Eu $4 f$ valence band XPS spectra of EuO films on YSZ (001), grown at $400^{\circ} \mathrm{C}$ with a $8.2 \AA / \mathrm{min}$ Eu flux rate for 10 min. The spectra were collected at normal emission. From top to bottom: EuO films grown under oxygen pressures of $16,12,10,8$, and $4 \times 10^{-8}$ mbar, and clean YSZ substrate.

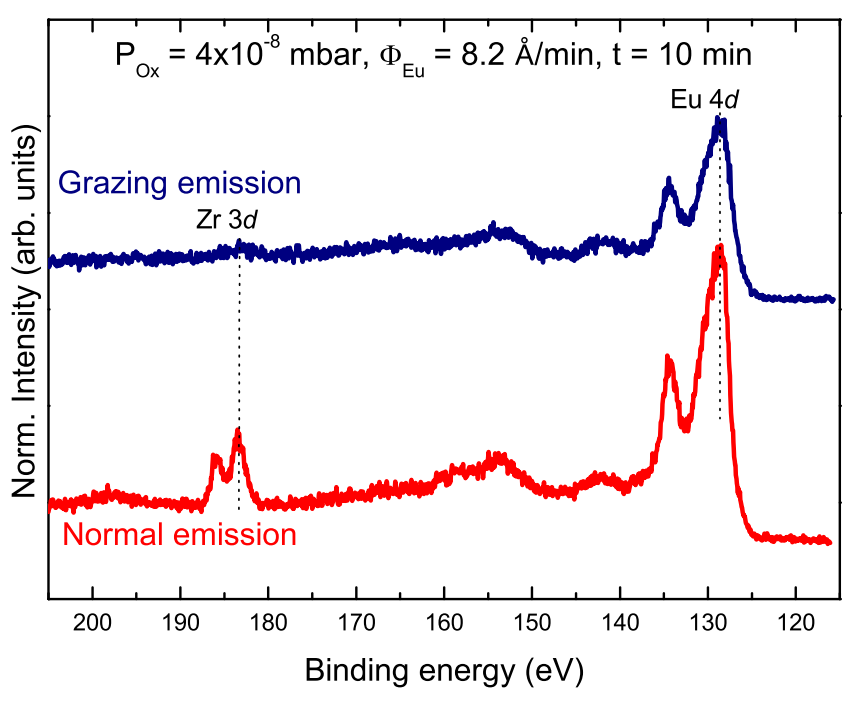

FIG. 7: (Color online) Take-off angle dependence of the $\mathrm{Zr} 3 d-\mathrm{Eu} 4 d$ core level XPS spectra of a EuO film on YSZ (001). Top: grazing emission, i.e., $\Theta=70^{\circ}$ with respect to the surface normal. Bottom: normal emission. The film was grown at $400^{\circ} \mathrm{C}$ under a $4 \times 10^{-8}$ mbar oxygen pressure and a $8.2 \AA / \mathrm{min}$ Eu flux rate for $10 \mathrm{~min}$.

fectly free from $\mathrm{Eu}^{3+}$ species. In case oxygen is present in the MBE chamber, YSZ is supplying only the amount of oxygen that is needed to complete the formation of EuO. This also explains why the growth rate of the first five to six monolayers is determined only by the Eu flux rate and is totally independent of the supply of oxygen pressure in the MBE chamber, see Fig. 2. It is important that the pressure is kept below the critical value of
$10-12 \times 10^{-8}$ mbar as we will discuss in more detail in Sec. IV

Based on the comprehensive set of RHEED, LEED, and XPS data, including the RHEED intensity oscillations, we have now demonstrated that $\mathrm{EuO}$ thin films can be grown epitaxially in a layer-by-layer fashion with good control of its chemical state. The supply of oxygen from the YSZ does not do any harm, and in fact, it can be utilized as a welcoming method to calibrate the Eu flux rate accurately, e.g., the $8.1-8.2 \AA / \mathrm{min}$ from the quartz crystal monitor corresponds to the growth of one monolayer EuO per $25 \mathrm{~s}$.

\section{SUSTAINED GROWTH OF EuO ON YSZ}

Having shown that the initial stages of growth of EuO on YSZ (001) can be made quite perfect, we now investigate whether thicker EuO films can be prepared while keeping the epitaxy and, especially, the layer-by-layer growth mode. We therefore have grown films for longer deposition times, e.g., between 100 and 200 min, using a series of finely intervalled pressures for the oxygen, e.g., $4,8,10,12$, and $16 \times 10^{-8}$ mbar. The Eu flux rates were kept at $8.0-8.3 \AA / \mathrm{min}$. The resulting thickness of the films varies between roughly 300 and $800 \AA$ as will be discussed later. The RHEED and LEED results are plotted in Fig. 8. One can clearly see that excellent epitaxial growth has been achieved for (a) 4, (b) 8, and (c) $10 \times 10^{-8}$ mbar oxygen pressures. For $12 \times 10^{-8}$ mbar (d) or higher pressures, however, the appearance of additional spots in the RHEED indicates that the surface structure starts to change, and the absence of a pattern in 

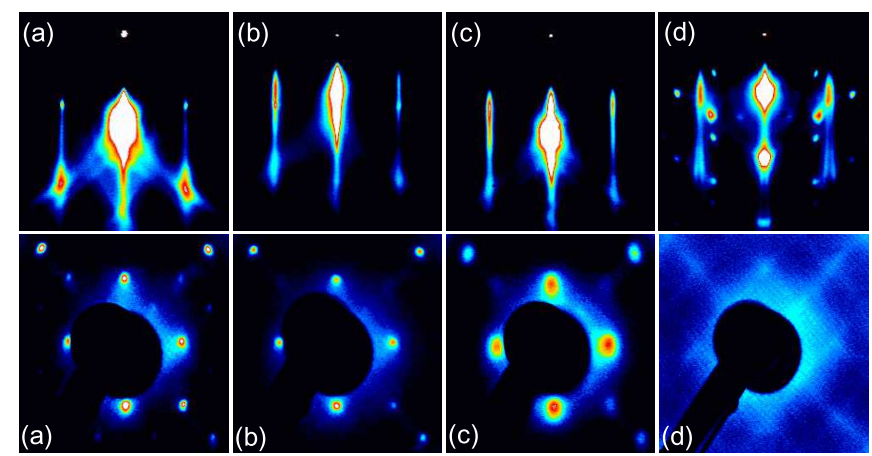

FIG. 8: (Color online) Top panels: RHEED photographs of $\mathrm{EuO}$ films on $\mathrm{YSZ}(001)$ grown at $400^{\circ} \mathrm{C}$ with $8.0-8.3 \AA / \mathrm{min}$ $\mathrm{Eu}$ flux rates under oxygen pressures of - from left to right - (a) 4, (b) 8, (c) 10, and (d) $12 \times 10^{-8}$ mbar. The deposition times were 200, 200, 100, and $100 \mathrm{~min}$, respectively. The RHEED electron energy was $20 \mathrm{keV}$ with the beam incident along the [100] direction. Bottom panels: corresponding LEED photographs. The LEED electron energies were 369, 368,370 , and $266 \mathrm{eV}$, respectively.

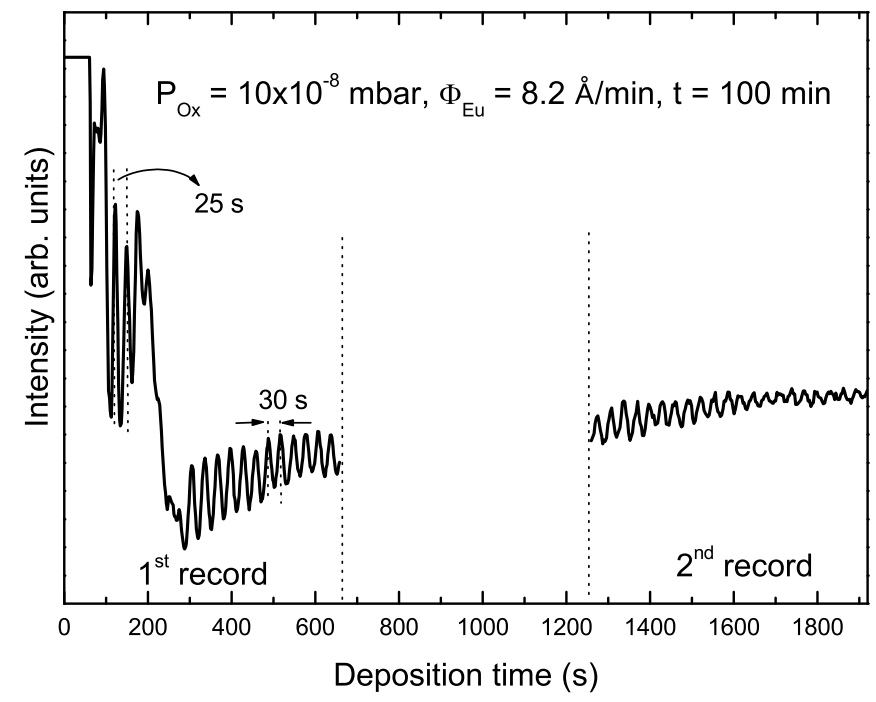

FIG. 9: RHEED intensity oscillations of the specularly reflected electron beam, recorded during deposition of a $\mathrm{EuO}$ film on YSZ (001) grown at $400^{\circ} \mathrm{C}$ using a $10 \times 10^{-8} \mathrm{mbar}$ oxygen pressure and a $8.2 \AA / \mathrm{min}$ Eu flux rate.

the LEED even suggests appreciable surface roughness. The LEED photographs were taken at electron beam energies of 368-370 eV since lower energies did not provide stable patterns due to charging.

As discussed in Sec III, the initial five to six oscillations of the specular reflected RHEED beam intensity are a unique feature for the initial stages of $\mathrm{EuO}$ growth on the YSZ (001). These initial oscillations do always occur, i.e., independent of the oxygen pressure in the MBE chamber, unless the pressure exceeds a critical value above which trivalent Eu species are formed. Remarkable is that no more oscillations can be observed beyond these five to

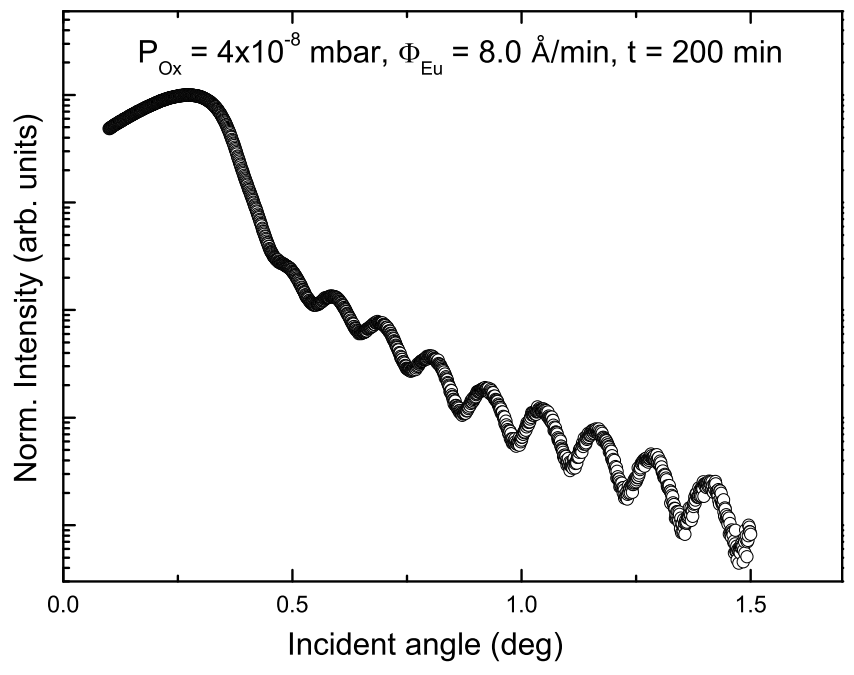

FIG. 10: XRR curve of epitaxial EuO film on YSZ (001) grown for $200 \mathrm{~min}$ at $400^{\circ} \mathrm{C}$ using a $4 \times 10^{-8}$ mbar oxygen pressure and a $8.0 \AA / \mathrm{min} \mathrm{Eu}$ flux rate.

six when growing thicker films. This is the case for a wide range of oxygen pressures. There is one exception: for a pressure of $10 \times 10^{-8} \mathrm{mbar}$, we were able to see further RHEED intensity oscillations. Figure 9 shows that the initial five to six oscillations are then followed by at least 50 more oscillations. It demonstrates that a layer-by-layer growth mode for $\mathrm{EuO}$ is possible during the sustained growth. Interestingly, the oscillation period during the sustained growth is similar and yet a little bit larger than during the initial stages of growth: 30 $\mathrm{s}$ against $25 \mathrm{~s}$. Apparently, the oxygen pressure must be close to and yet a little less than the critical value in order to maintain the layer-by-layer growth mode, while the Eu flux determines the growth rate in the initial stages, i.e. $8.0-8.2 \AA / \mathrm{min}$ Eu flux corresponding to $25 \mathrm{~s}$. per EuO layer, it is the limited oxygen supply from the MBE environment which dictates the speed during the sustained growth, i.e., to $30 \mathrm{~s}$ per EuO layer.

To elucidate further the growth process, we have measured the thickness of the films using ex situ XRR measurements. Since $\mathrm{EuO}$ deteriorates rapidly under ambient conditions, the films need to be capped. To this end, an aluminum layer with a thickness of 20-40 $\AA$ has been evaporated on top of the EuO. This thickness turns out to be sufficient for the aluminum to be a good protective overlayer as will be discussed later. Figure 10 exhibits the XRR profile of the $\mathrm{EuO}$ film which was grown at an oxygen pressure of $4 \times 10^{-8} \mathrm{mbar}$ for $200 \mathrm{~min}$. The corresponding RHEED and LEED patterns of the EuO film are displayed in Fig. 8(a). From the period of interference fringes, we deduce that the thickness of the $\mathrm{EuO}$ film is about $350 \AA$.

Thicknesses of the other films are also determined from their XRR profiles. The results are displayed in Fig. 11, where we plot the thickness against the product of oxygen pressure and total deposition time. For oxygen pressure 


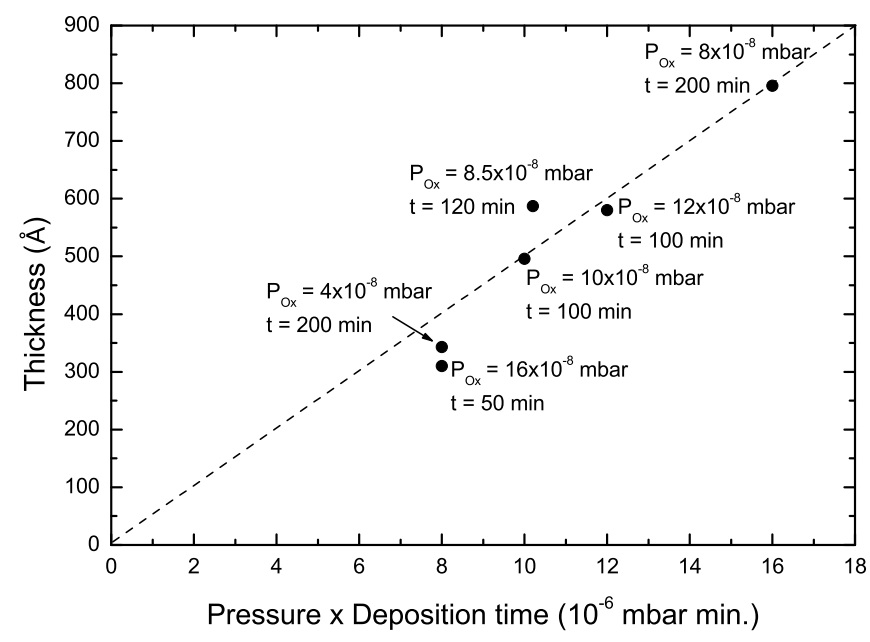

FIG. 11: EuO film thickness, as determined from XRR measurements, versus the product of oxygen pressure and total deposition time.

up to $12 \times 10^{-8}$ mbar we can observe a clear and direct linear relationship between them, strongly suggesting that the thickness is determined by the amount of oxygen incorporated. In other words, the growth is limited by the amount of oxygen made available. This in turn means that the Eu flux rate is higher than necessary and that the excess Eu must be re--evaporated into the vacuum. Figure 11 essentially confirms the distillation process needed to maintain good control of the stoichiometry as reported in our earlier studies by Steeneken et $a l .8,28$ and Tjeng et al. . $^{33}$

We now investigate to what extent the growth conditions affect the magnetic properties of the $\mathrm{EuO}$ films using a SQUID magnetometer. The results are shown in Fig. 12. The films grown with $4,8,8.5$, and $10 \times 10^{-8}$ mbar oxygen pressures all have a Curie temperature of 69 $\mathrm{K}$ with a magnetic moment of $7 \mu_{B} /$ f.u. as expected for a $4 f^{7}$ system. The inset shows the field dependence of the magnetization at $5 \mathrm{~K}$ for the film grown with a $8 \times 10^{-8}$ mbar oxygen pressure. Here one can observe a hysteresis behavior with a saturation magnetization of $7 \mu_{B}$. These results are in agreement with the RHEED and LEED results as displayed in Figs. 8(a)-8(c), in the sense that the proper ferromagnetic properties are always maintained as long as good epitaxial growth is also achieved. On the other hand, films grown with 12 and $16 \times 10^{-8}$ mbar have completely lost their ferromagnetic properties. It is remarkable that exceeding the $10 \times 10^{-8}$ mbar value just a little bit causes such a dramatic change. This very abrupt change is also consistent with the RHEED and LEED results as displayed in Fig. 8 (d). Considerable film roughness starts to develop for oxygen pressures higher than $10 \times 10^{-8}$ mbar. We would like to infer that having only a $T_{C}$ of about $69 \mathrm{~K}$ is not a sufficient characteristic to conclude that the film is homogeneous and stoichiometric. One also needs to establish that the film has a full saturation magnetization of $7 \mu_{B}$. The measure-

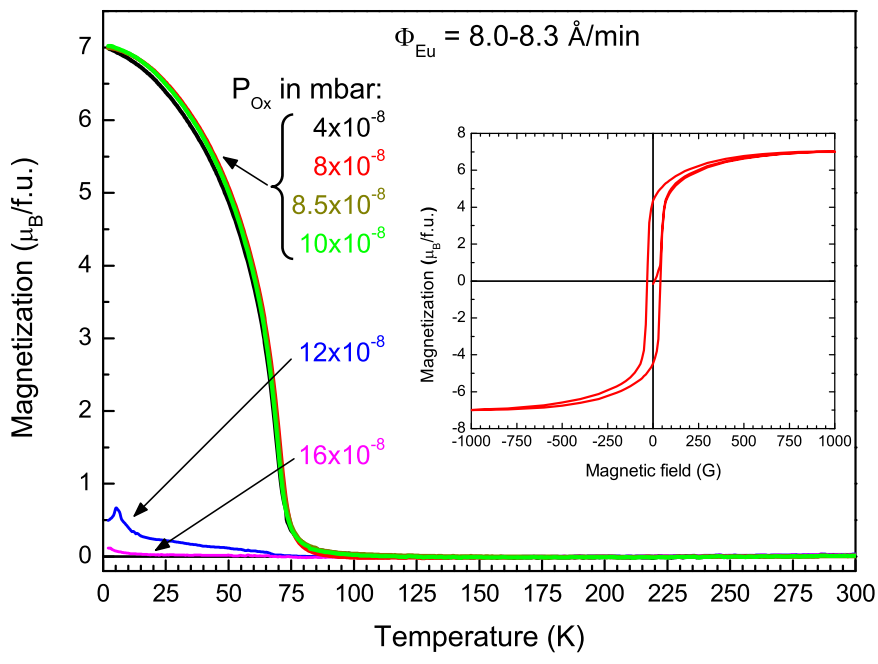

FIG. 12: (Color online) Temperature dependence of the magnetization of epitaxial $\mathrm{EuO}$ films on YSZ (001) grown at

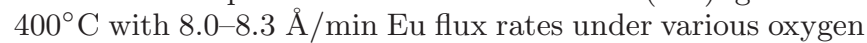
pressures as indicated. The small magnetization contribution from the substrate has been subtracted. The applied magnetic field was $1000 \mathrm{G}$. The inset shows the field dependence of the magnetization of epitaxial $\mathrm{EuO}$ on YSZ (001) at $5 \mathrm{~K}$. The film was grown at $400^{\circ} \mathrm{C}$ with a $8.2 \AA / \mathrm{min}$ Eu flux rate and a $8 \times 10^{-8}$ mbar oxygen pressure.

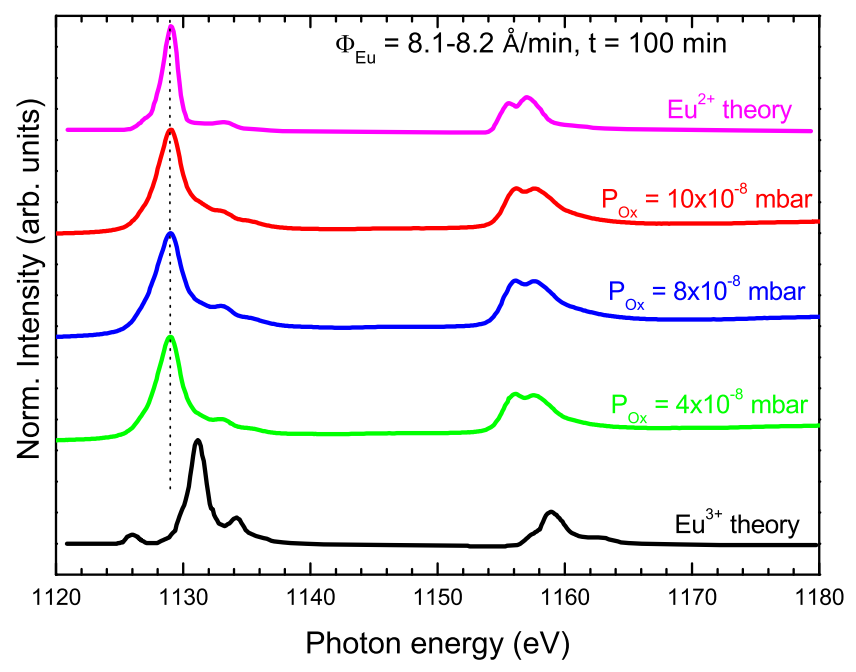

FIG. 13: (Color online) Eu $M_{4,5}(3 d \rightarrow 4 f)$ XAS spectra of EuO films grown epitaxially on YSZ (001) at $400^{\circ} \mathrm{C}$ with 8.1-8.2 $\AA / \mathrm{min}$ Eu flux rates under various oxygen pressures as indicated. The films are capped with a $20-40 \AA$ aluminum overlayer. Theoretical spectra of $\mathrm{Eu}^{2+}$ and $\mathrm{Eu}^{3+}$ are also shown, retrieved from Ref. [46].

ment of the magnetic properties can therefore serve as a critical test for the growth conditions and in particular, the oxygen stoichiometry of the EuO films.

We have also performed ex situ soft XAS measurements at the Eu $M_{4,5}$ edges to examine the integrity of the EuO films after capping with the aluminum overlayer. Figure 13 depicts the XAS spectra together with the the- 
oretical spectra for $\mathrm{Eu}^{2+}$ (top) and $\mathrm{Eu}^{3+}$ (bottom) It is clear that the Eu spectra are very similar to the theoretical spectrum for $\mathrm{Eu}^{2+}$, meaning that the $\mathrm{EuO}$ films with 4,8 , and $10 \times 10^{-8}$ mbar oxygen pressures are completely free from $\mathrm{Eu}^{3+}$ species. This in turn implies that an aluminum overlayer as thin as 20-40 $\AA$ works well to protect the $\mathrm{EuO}$ films against air, contrary to the claims made elsewhere that one needs very thick capping layers, $26,27,29,30,31,32,34,36,38,39$ We attribute this to the fact that the epitaxial growth of EuO on YSZ (001) yields such a smooth film so that a very thin aluminum film is sufficient to make a closed capping overlayer. The smoothness of the films as well as the complete absence of $\mathrm{Eu}^{3+}$ impurities forms a good starting point for the fabrication of well-defined interfaces with other metals or oxide materials, thereby opening up new opportunities to study or even generate new phenomena related to interface physics.

We conclude that the critical oxygen pressure is around $10-12 \times 10^{-8} \mathrm{mbar}$ for a $8.0-8.3 \AA / \mathrm{min}$ Eu flux rate. Only below this pressure one has the distillation process taking place so that good epitaxial growth can be achieved with the proper stoichiometry and ferromagnetic properties. Apparently, layer-by-layer growth can be obtained only if one is close to, but not exceeding, the critical pressure.

\section{EPITAXIAL GROWTH OF EuO ON MgO}

Having understood the growth process of EuO on YSZ (001), and having found the recipe to obtain excellent epitaxial growth of EuO on YSZ (001), we now turn our attention to the preparation of $\mathrm{EuO}$ on $\mathrm{MgO}$ (001). $\mathrm{MgO}$ as a substrate for EuO is interesting since several studies $25,27,28,35$ have reported good epitaxial growth despite the very large lattice mismatch of about $20 \%$. The RHEED and LEED photographs of EuO films grown on $\mathrm{MgO}$ using different oxygen pressures are displayed in Fig. 14. EuO films with oxygen pressure below $10 \times 10^{-8}$

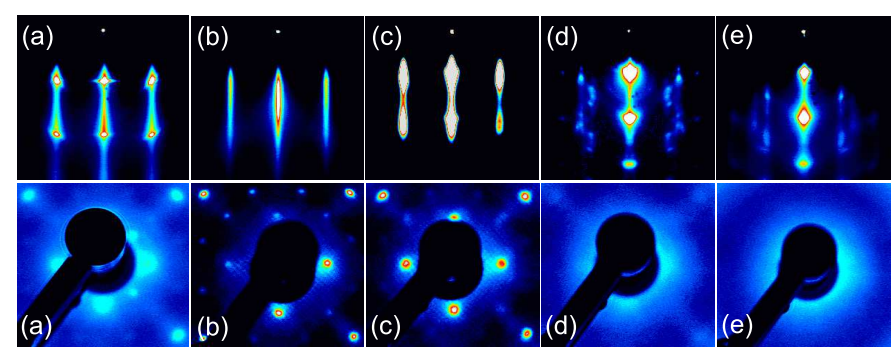

FIG. 14: (Color online) Top panels: RHEED photographs of $\mathrm{EuO}$ films on $\mathrm{MgO}(001)$ grown at $400^{\circ} \mathrm{C}$ with $8.1-8.2 \AA / \mathrm{min}$ $\mathrm{Eu}$ flux rates under oxygen pressures of - from left to right (a) 4 , (b) 8 , (c) 10, (d) 12 , and (e) $16 \times 10^{-8}$ mbar. The deposition times were all $100 \mathrm{~min}$. The RHEED electron energy was $20 \mathrm{keV}$ with the beam incident along the [100] direction. Bottom panels: corresponding LEED photographs. The LEED electron energy was set at $360 \mathrm{eV}$.

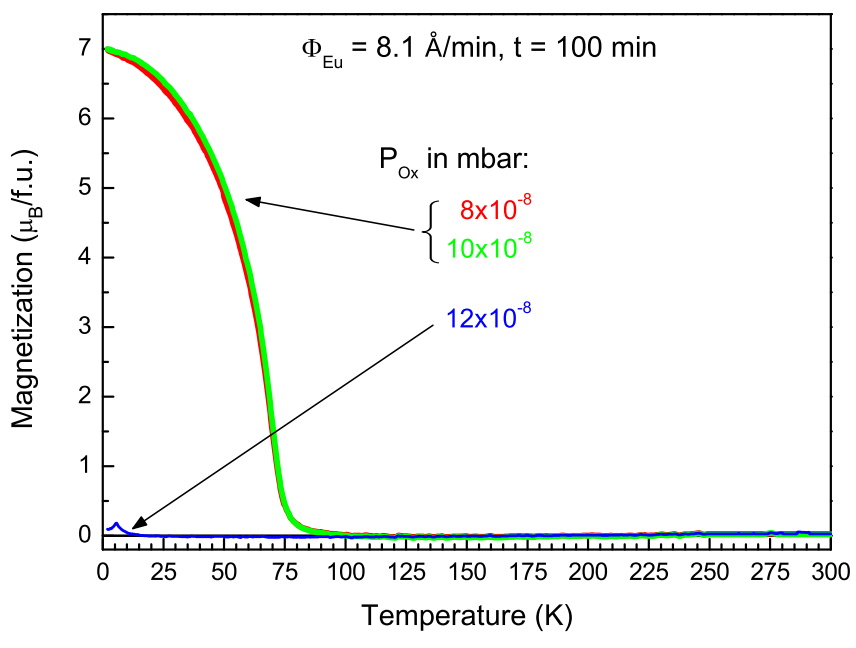

FIG. 15: (Color online) Temperature dependence of the magnetization of epitaxial EuO films on $\mathrm{MgO}$ (001) grown at $400^{\circ} \mathrm{C}$ with a $8.1 \AA / \mathrm{min} \mathrm{Eu}$ flux rate under various oxygen pressures as indicated. The small magnetization contribution from the substrate has been subtracted. The applied magnetic field was $1000 \mathrm{G}$.

mbar all show excellent epitaxial growth. The relationship between the distances of the $\mathrm{MgO}$ and $\mathrm{EuO}$ streaks is consistent with the ratio of their lattice constants of 1.22. On the other hand, a higher oxygen pressure creates additional spots in the RHEED image and even causes the LEED pattern to disappear. Similar to the EuO on YSZ case, this indicates that $10-12 \times 10^{-8}$ is the critical value for the oxygen pressure above which one no longer gets crystalline and stoichiometric EuO.

Our attempts to observe RHEED intensity oscillations of EuO films grown on $\mathrm{MgO}$ (001) were yet unsuccessful. The RHEED streaks and specular spot suddenly disappeared right after the deposition of EuO has been initiated. In approximately $30 \mathrm{~s}$, new streak lines appear, whose spacing conforms to the EuO lattice parameter. However, the specular spot was never recovered. A more detailed growth study is required to determine the growth process of $\mathrm{EuO}$ on $\mathrm{MgO}$ (001).

To investigate the quality of these EuO films in terms of their magnetic properties, we have performed SQUID measurement for films grown with oxygen pressures of 8,10 , and $12 \times 10^{-8}$ mbar. The results are shown in Fig. 15. Similar to the EuO on YSZ case, the films that were grown below $10 \times 10^{-8}$ mbar have a Curie temperature of $69 \mathrm{~K}$ with a magnetic moment close to $7 \mu_{B} /$ f.u. Conversely, the film grown under a higher oxygen pressure has completely lost its ferromagnetic character. The small peak at roughly $5 \mathrm{~K}$ indicates the typical antiferromagnetic ordering temperature of $\mathrm{Eu}_{3} \mathrm{O}_{4}$, meaning that the dramatic loss of the ferromagnetism for the growth using slightly above the critical oxygen pressure is due to the presence of $\mathrm{Eu}^{3+}$ species. 


\section{CONCLUSION}

We have successfully grown epitaxial and highly stoichiometric EuO films on YSZ (001). The initial stages of growth involve the limited supply of oxygen from the YSZ substrate, but the EuO stoichiometry can still be well maintained. We have also observed RHEED intensity oscillations during the sustained stages of growth, which demonstrate that the layer-by-layer growth mode can be achieved for EuO films on YSZ (001). The EuO films were sufficiently smooth so that capping with a thin layer of aluminum enabled ex situ experiments free from trivalent Eu species. The excellent epitaxial growth of EuO films is always accompanied by equally excellent ferromagnetic properties: a saturation magnetization of $7 \mu_{B}$ and a $T_{C}$ of $69 \mathrm{~K}$. We have also confirmed that the use of the Eu-distillation process during the MBE-assisted growth enables the consistent achievement of stoichiometry. The layer-by-layer growth, the smoothness of the film, and the excellent stoichiometry provide an excellent basis for the fabrication of well--defined interfaces for further studies.

\section{ACKNOWLEDGMENTS}

We would like to thank Lucie Hamdan for her skillful technical and organizational assistance in preparing the experiment. We acknowledge the NSRRC staff for providing us with beam time. The research in Cologne is supported by the Deutsche Forschungsgemeinschaft through SFB 608.
1 For a review, see A. Mauger and C. Godart, Phys. Rep. 141, 51 (1986).

2 M. R. Oliver, J. O. Dimmock, A. L. McWhorter, and T. B. Reed, Phys. Rev. B 5, 1078 (1972).

3 Y. Shapira, S. Foner, and T. B. Reed, Phys. Rev. B 8, 2299 (1973).

${ }^{4}$ Y. Shapira, S. Foner, R. L. Aggarwal, and T. B. Reed, Phys. Rev. B 8, 2316 (1973).

5 A. Mauger, C. Godart, M. Escorne, J. C. Achard, and J. P. Desfours, J. Phys. (Paris) 39, 1125 (1978).

6 D. DiMarzio, M. Croft, N. Sakai and M. W. Shafer, Phys. Rev. B 35, 8891 (1987).

7 M. M. Abd-Elmeguid and R. D. Taylor, Phys Rev. B 42, 1048 (1990).

8 P. G. Steeneken, L. H. Tjeng, I. Elfimov, G. A. Sawatzky, G. Ghiringhelli, N. B. Brookes, and D.-J. Huang, Phys. Rev. Lett. 88, 047201 (2002).

${ }^{9}$ K. Y. Ahn and J. C. Suits, IEEE Trans.Magn. 3, 453 (1967).

${ }^{10}$ K. Y. Ahn and T. R. McGuire, J. Appl. Phys. 39, 5061 (1968).

11 K. Y. Ahn and M. W. Shafer, J. Appl. Phys. 41, 1260 (1970).

12 K. Lee and J. C. Suits, J. Appl. Phys. 41, 954 (1970).

13 K. Lee and J. C. Suits, Phys. Lett. 34A, 141 (1971).

14 K. Y. Ahn, K. N. Tu, and W. Reuter, J. Appl. Phys. 42, 1769 (1971).

15 T. R. McGuire, G. F. Petrich, B. L. Olson, V. L. Moruzzi, and K. Y. Ahn, J. Appl. Phys. 42, 1775 (1971).

16 J. C. Suits, K. Lee, H. F. Winters, P. B. P. Phipps, and D. F. Kyser, J. Appl. Phys. 42, 1777 (1971).

17 J. C. Suits and K. Lee, J. Appl. Phys. 42, 3258 (1971).

18 C. Paparoditis, R. Suryanarayanan, C. Llinares, E. Monteil, and G. Bordure, Solid State Commun. 9, 1871 (1971).

19 C. Llinares, E. Monteil, G. Bordure, and C. Paparoditis, Solid State Commun. 13, 205 (1973).

20 C. Llinares, C. Duchemin, and G. Bordure, Solid State Commun. 13, 785 (1973).

21 O. Massenet, Y. Capiomont, and N. V. Dang, J. Appl. Phys. 45, 3593 (1974).

22 C. Llinares, L. Gouskov, C. Duchemin, and G. Bordure, J. Phys. Chem. Solids 36, 567 (1975).
${ }^{23}$ M. W. Shafer, J. B. Torrance, and T. Penney, J. Phys. Chem. Solids 33, 2251 (1972).

24 G. M. Roesler, Jr., M. E. Filipkowski, P. R. Broussard, Y. U. Idzerda, M. S. Osofsky, and R. J. Soulen, Jr., in Superconducting Superlattices and Multilayers, edited by I. Bozovic, (SPIE Bellingham, 1994), Vol. 2517, pp. 285-290.

25 M. Sohma, K. Kawaguchi, and Y. Oosawa, J. Appl. Phys. 81, 5301 (1997).

26 N. Iwata, T Morishita, and K. Kohn, J. Phys. Soc. Japan 69, 1745 (2000).

27 N. Iwata, G. Pindoria, T Morishita, and K. Kohn, J. Phys. Soc. Japan 69, 230 (2000).

28 P. G. Steeneken, Ph.D. thesis, University of Groningen, 2002.

29 J. Lettieri, V. Vaithyanathan, S. K. Eah, J. Stephens, V. Sih, D. D. Awschalom, J. Levy, and D. G. Schlom, Appl. Phys. Lett. 83, 975 (2003).

30 T. Matsumoto, K. Yamaguchi, M. Yuri, K. Kawaguchi, N. Koshizaki, and K. Yamada, J. Phys.: Condens. Matter. 16, 6017 (2004).

31 J. Holroyd, Y. U. Idzerda, and S. Stadler, J. Appl. Phys. 95, 6571 (2004).

32 T. S. Santos and J. S. Moodera, Phys. Rev. B 69, 241203(R) (2004).

${ }^{33}$ H. Ott, S. J. Heise, R. Sutarto, Z. Hu, C. F. Chang, H. H. Hsieh, H.-J. Lin, C. T. Chen, and L. H. Tjeng, Phys. Rev. B 73, 094407 (2006).

34 E. Negusse, J. Holroyd, M. Liberati, J. Dvorak, Y. U. Idzerda, T. S. Santos, J. S. Moodera, and E. Arenholz , J. Appl. Phys. 99, 08E507 (2006).

35 H. Lee, J.-Y. Kim, K.-J. Rho, B.-G. Park, and J.-H. Park, J. Appl. Phys. 102, 053903 (2007).

36 A. Schmehl, V. Vaithyanathan, A. Herrnberger, S. Thiel, C. Richter, M. Liberati, T. Heeg, M. Röckerath, L. F. Kourkoutis, S. Mühlbauer, P. Böni, D. A. Muller, Y. Barash, J. Schubert, Y. Idzerda, J. Mannhart, and D. G. Schlom, Nat. Mater. 6, 882 (2007).

37 S. Mühlbauer, P. Böni, R. Georgii, A. Schmehl, D. G. Schlom, and J. Mannhart, J. Phys.: Condens. Matter. 20, 104230 (2008).

38 G. van der Laan, E. Arenholz, A. Schmehl, and D. G. Schlom, Phys. Rev. Lett. 100, 067403 (2008). 
${ }^{39}$ R. W. Ulbricht, A. Schmehl, T. Heeg, J. Schubert, and D. G. Schlom, Appl. Phys. Lett. 93, 102105 (2008).

40 R. P. Panguluri, T. S. Santos, E. Negusse, J. Dvorak, Y. Idzerda, J. S. Moodera, and B. Nadgorny, Phys. Rev. B 78, 125307 (2008).

${ }^{41}$ N. J. C. Ingle and I. S. Elfimov, Phys. Rev. B 77, 121202(R) (2008).

42 R. P. Ingel and D. Lewis III, J. Am. Ceram. Soc. 69, 325 (1986).

43 M. Yashima, S. Sasaki, M. Kakihana, Y. Yamaguchi, H. Arashi, and M. Yoshimura, Acta Cryst. B50, 663 (1994).
${ }^{44}$ V. E. Henrich and P. A. Cox, The Surface Science of Metal Oxides (Cambridge University Press, 1994). Table 2.2.

45 N. Q. Minh, J. Am. Ceram. Soc. 76, 563 (1993).

46 B. T. Thole, G. van der Laan, J. C. Fuggle, G. A. Sawatzky, R. C. Karnatak, and J.-M. Esteva, Phys. Rev. B 32, 5107 (1985).

47 J. B. Goedkoop, B. T. Thole, G. van der Laan, G. A. Sawatzky, F. M. F. de Groot, and J. C. Fuggle, Phys. Rev. B 37, 2086 (1988). 